\title{
Characteristics and early outcomes of children and adolescents treated with darunavir/ritonavir-, raltegravir- or etravirine-containing antiretroviral therapy in the Western Cape Province of South Africa
}

\author{
J Nuttall, MB ChB, MSc; V Pillay, MB ChB, MPH \\ Paediatric Infectious Diseases Unit, Department of Paediatrics and Child Health, Faculty of Health Sciences, University of Cape Town and \\ Red Cross War Memorial Children's Hospital, Cape Town, South Africa
}

Corresponding author: J Nuttall (james.nuttall@uct.ac.za)

\begin{abstract}
Background. There is an increasing need for third-line treatment regimens in HIV-infected children with antiretroviral treatment (ART) failure. Data are limited on darunavir/ritonavir (DRV/r)-, raltegravir (RAL)- and etravirine (ETR)-containing regimens in treatmentexperienced children from resource-constrained settings receiving these drugs as part of routine care.

Objective. To describe the characteristics and early outcomes of treatment-experienced children ( $<20$ years of age) in the Western Cape Province of South Africa treated with DRV/r-, RAL- or ETR-containing regimens.

Methods. This was a retrospective review of treatment-experienced children receiving a DRV/r-, RAL- or ETR-containing regimen as recommended by a paediatric expert review committee, based on HIV drug resistance testing.

Results. Thirty-five children of median age 8.8 years (interquartile range (IQR) 5.5 - 11) who had received ART for a median of 6.9 years (IQR 5 - 9.9) and started a DRV/r-, RAL- or ETR-containing regimen were included. Before starting such a regimen, the median CD4+ lymphocyte count and HIV-1 RNA level were 405.5 cells/ $\mu$ L (IQR 251.5 - 541) and 28314 copies/mL (IQR 5595.5 - 120 186.5) (log 4.5 (IQR 3.7 - 5)), respectively, in 24 subjects with available results. After a median of 2 years (IQR 1.3 - 4) on treatment, 29/30 (96.7\%) and 23/30 (76.7\%) subjects with available results had HIV-1 RNA levels of $<400$ and $<50$ copies/mL, respectively.

Conclusions. This study found DRV/r-, RAL- and ETR-containing regimens to be effective in a group of treatment-experienced children and adolescents with multidrug-resistant HIV. Although the treatment regimens in this study were individualised based on HIV genotyping results, further research evaluating the safety and efficacy of standardised third-line treatment regimens in children of all ages is needed.

S Afr Med J 2018;108(2):105-110. DOI:10.7196/SAMJ.2018.v108i2.12573
\end{abstract}

By 2012, the estimated number of children (0 - 14 years of age) receiving antiretroviral therapy (ART) in South Africa (SA) was 140 541, representing an estimated $63 \%$ of those requiring treatment. ${ }^{[1,2]}$ As increasing numbers of children are started on first- and second-line ART, the demand for third-line regimens in children and adolescents with treatment failure is likely to increase. It is estimated that currently $<1 \%$ of people on ART globally are receiving third-line regimens, and it is unknown what proportion of these are children. ${ }^{[3]}$

An unpublished systematic review presented in 2015 assessing second- and third-line ART options for children and adolescents concluded that there is insufficient evidence to directly evaluate alternative second- and third-line ART options for children, and that current recommendations are still based on inference from adult trials. ${ }^{[4]}$ The World Health Organization (WHO) recommends that national programmes should develop policies for third-line ART that should incorporate integrase strand transfer inhibitors (INSTIs), second-generation protease inhibitors (PIs) and secondgeneration non-nucleoside reverse transcriptase inhibitors (NNRTIs) with minimal risk of cross-resistance to previously used regimens. Recommended third-line ART regimens in the WHO 2016 guidelines ${ }^{[5]}$ are: (i) darunavir/ritonavir (DRV/r) plus dolutegravir (DTG) (or raltegravir (RAL)) with or without one to two nucleoside reverse transcriptase inhibitors (NRTIs); (ii) DRV/r plus two NRTIs with or without one NNRTI; or (iii) RAL (or DTG) plus two NRTIs, depending on the preceding first- and second-line ART regimens.

Although safety and efficacy of DRV/r, etravirine (ETR) and RAL have been reported in specific age groups of ART-naive and ARTexperienced children and adolescents, there is a paucity of data on treatment-experienced children from resource-constrained settings receiving these drugs as part of routine care ${ }^{[6-14]}$ In addition, DRV/r is not recommended in children $<3$ years of age owing to toxicity concerns in animal studies, and ETR, a second-generation NNRTI, is not recommended in children $<6$ years of age owing to lack of safety and efficacy data. Until very recently, DTG was only recommended by the US Food and Drug Administration (FDA) for adolescents $>12$ years of age and $>40 \mathrm{~kg}$ body weight. ${ }^{[15]}$ The FDA has recently approved DTG from 6 to $<12$ years and $\geq 30 \mathrm{~kg}$ in a dose of $35 \mathrm{mg}$ once daily, but suitable formulations that allow for administration of this dose are not yet registered in SA. ${ }^{[16]}$ In SA, DTG has only been approved from 18 years of age.

\section{Objective}

To describe the characteristics and early outcomes of treatmentexperienced children and adolescents $(<20$ years of age $)$ in the Western Cape Province of SA who initiated an ART regimen that included one or more of DRV/r, RAL and ETR. 


\section{Methods}

The Western Cape paediatric ART expert review committee (ERC) was constituted in October 2013. Clinicians managing children and adolescents with antiretroviral (ARV) drug resistance on genotype resistance testing (GRT) submitted a standardised application form requesting guidance on further treatment to the ERC. The main eligibility criterion for treatment with DRV/r, RAL or ETR was the presence of PI resistance on GRT, defined as a lopinavir/ritonavir $(\mathrm{LPV} / \mathrm{r})$ or atazanavir/ritonavir (ATV/r) mutation score (MS) of $\geq 15$ using the Stanford University HIV genotypic resistance interpretation algorithm. ${ }^{[17]}$

Individualised treatment recommendations for patients were made on a consensus basis by the ERC taking into account age, weight, previous ARV exposure, concomitant medication, availability of paediatric formulations of newer-generation ARV drugs, and an assessment of adherence to medication provided by the applicant. In April 2015, a consensus treatment algorithm based on local and international expert opinion, available data and international guidelines was adopted by the ERC in an attempt to standardise recommendations based on patterns of ARV drug resistance and MSs. According to this algorithm, all patients with an LPV/r or $\mathrm{ATV} / \mathrm{r}$ MS of $\geq 15$ would receive $\mathrm{DRV} / \mathrm{r}$ plus either lamivudine (3TC) or emtricitabine (FTC), and either zidovudine (AZT) or abacavir (ABC) depending on which had the lowest MS. Tenofovir (TDF) was considered in patients aged $>12$ years and weighing $>40 \mathrm{~kg}$ with normal renal function. Indications for the inclusion of RAL included low-level or higher resistance to DRV/r $(\mathrm{MS} \geq 15)$ or no active NRTIs being available (ABC, AZT and TDF MS $\geq 30$ ). ETR was considered for inclusion in the regimen in addition to RAL if the genotype indicated low-level or higher resistance to DRV/r, no active NRTIs were available and the ETR MS was $<30$, particularly if there was no known previous exposure to NNRTIs.

Children and adolescents who were already receiving ARV regimens containing DRV/r, RAL or ETR prior to the establishment of the provincial ERC were also reviewed by the ERC and included in the study database. These patients were identified by the Western Cape HIV/AIDS, sexually transmitted infections and tuberculosis (HAST) pharmaceutical policy specialist because they were receiving drugs that were not included on the provincial pharmaceutical code list at the time. The clinicians managing these patients were requested by the HAST pharmaceutical policy specialist to submit application forms and HIV resistance tests to the Western Cape ERC motivating for the continued use of these ARV regimens.

Children and adolescents were managed at a wide spectrum of healthcare facilities ranging from primary care clinics to district, regional and tertiary hospitals by the attending clinician who submitted the application to the ERC. There was no standardised adherence support or follow-up by specialists other than what was routinely provided at the healthcare facility where the patient was managed. Recommended monitoring was according to provincial guidelines: HIV-1 RNA after 6 and 12 months on treatment and then annually if suppressed or 3 - 6-monthly if not suppressed, CD4+ lymphocyte count annually until $>200$ cells $/ \mu \mathrm{L}$, and monitoring of serum creatinine and full blood count recommended in patients on TDF and AZT, respectively. ${ }^{[18]}$

The data sources for this study comprised the following: application forms and HIV resistance test results, including Stanford University HIV genotypic resistance interpretation results performed at the time of the application, submitted by referring clinicians to the Western Cape ERC; ARV treatment recommendations by the ERC; and available laboratory test results following initiation of the recommended ARV regimens. The lead author (JN) is a member of the ERC and accessed the data from the electronic submissions to the ERC that are emailed to the ERC members by the Western Cape HAST pharmaceutical policy specialist.

The following data on treatment-experienced children and adolescents $(<20$ years of age at the time of application $)$ who initiated a DRV/r-, RAL- or ETR-containing ART regimen prior to October 2016 were entered by one of the authors (VP) into a study database: demographic and HIV disease characteristics, previous ARV exposure, spectrum of HIV drug resistance mutations, ARV treatment regimens approved by the ERC, and early treatment outcomes. For the purposes of this study, the following definitions were used: a first-line treatment regimen refers to the first ART regimen (comprising three or more ARV drugs) that a child or adolescent was initiated on; a second-line treatment regimen refers to a single-class switch from NNRTI to PI or vice versa, regardless of NRTI changes; and a third-line treatment regimen refers to an ART regimen that includes one or more of the ARVs DRV/r, RAL and ETR, even though the child or adolescent may have received less than two, or three or more, previous ART regimens.

Data were coded using unique study number identifiers and stored in a secure password-protected study database accessible only to the study investigators. Data quality was ensured by the lead author (JN) manually checking data entry accuracy by comparing application forms and HIV resistance test results with the database.

A descriptive analysis was performed of the following patient characteristics at the time of application to the ERC: age, gender, level of healthcare facility at which the patient was being managed, CD4+ lymphocyte counts and/or percentages, HIV-1 RNA levels, and current and previous ARV regimens. In addition, ARV drug resistance mutations and MS (according to the Stanford University HIV genotypic resistance interpretation algorithm performed at the time that the genotypic resistance test/s were done or, if not done then, at the time of application to the $\mathrm{ERC}^{[17]}$ ) were analysed along with the ARV treatment recommendations made by the ERC. Mutations were not re-entered into a current version of the Stanford algorithm, and cumulative or composite genotypes were not performed.

Treatment outcome measures analysed were HIV-1 RNA levels and CD4+ lymphocyte counts/percentages measured within 12 months of commencing a third-line ART regimen and at the time of analysis. CD4+ lymphocyte counts and percentages as well as HIV-1 RNA levels were measured by the National Health Laboratory Service.

Standard measures of frequency were used to express data. Median and interquartile range (IQR) values were used to describe numerical variables, while frequencies and percentages were used to describe categorical variables. Data were analysed using the Stata release 12.0 statistical software package (StataCorp, USA).

The study was approved by the Departmental Research Committee, Department of Paediatrics and Child Health, University of Cape Town, and the Human Research Ethics Committee, Faculty of Health Sciences, University of Cape Town (ref. no. 259/2015).

\section{Results}

Between October 2013 and October 2016, 45 applications were reviewed. At the time of application, 13 children (28.9\%) were already receiving a DRV/r-containing ART regimen; 11 of them were also receiving RAL. No child was receiving ETR. Fourteen children $(31.1 \%)$ were receiving holding regimens (nine were on 3TC monotherapy and five on triple-NRTI regimens).

A further 22 children started a DRV/r-, RAL- or ETR-containing ART regimen between October 2013 and October 2016. The total 
study population comprised 35 children. The number of children starting a DRV/r-, RAL- or ETR-containing ART regimen each year from 2010 to 2016 was $1,4,3,2,4,13$ and 8 . A temporary holding regimen consisting of 3TC monotherapy or a triple-NRTI regimen was recommended by the ERC for the 10 children excluded from this analysis.

The characteristics of the 35 study participants receiving a DRV/r-, RAL- or ETR-containing ART regimen are shown in Table 1. Twelve of the 35 participants (34.3\%) had received only one previous ART regimen before starting on the DRV/r-, RAL- or ETR-containing regimen (all RTV- or LPV/r-based first-line ART, including singledrug substitutions from RTV to LPV/r, a single NRTI switch, or temporary 3TC monotherapy).

The proportion of participants with resistance to selected ARV drugs prior to starting treatment on a DRV/r-, RAL- or ETR-containing ART regimen is summarised in Fig. 1. Thirtytwo $(91.4 \%)$ and $31(88.6 \%)$ participants had an MS of $\geq 15$ to $\mathrm{LPV} / \mathrm{r}$ and ATV/r, respectively, using the Stanford University HIV genotypic interpretation algorithm. Eighteen participants (51.4\%) had mutations conferring low- $(n=17,48.6 \%)$ or intermediate-level $(n=1,2.9 \%)$ resistance to DRV/r. None had high-level resistance. The proportion of study subjects with each PI mutation, including DRV resistance-associated mutations, detected prior to starting treatment on a DRV/r-, RAL- or ETR-containing ART regimen is shown in Fig. 2. Sixteen participants $(45.7 \%)$ had mutations conferring low- $(n=6,17.1 \%)$, intermediate- $(n=8,22.9 \%)$ or high-level $(n=2$, $5.7 \%)$ resistance to ETR. Resistance testing did not include INSTI mutations, as none of the participants had been exposed to this class of drugs at the time of genotyping.

The DRV/r-, RAL- or ETR-containing ART regimens, median duration on treatment at the time of analysis and CD4+ and HIV-1 RNA outcomes are summarised in Table 2. Since not all participants had blood tests performed at 6 and/or 12 months on treatment, the outcomes are expressed as median (IQR) values within 12 months of starting treatment and at the time of the analysis. We were able to access significantly fewer CD4+ lymphocyte count results than HIV-1 RNA results, both within 12 months of starting treatment $(n=23)$ and at the time of analysis $(n=21)$. Among $32(91.4 \%)$ and $30(85.7 \%)$ participants with HIV-1 RNA results available within 12 months of starting treatment and at a median of 2 years (IQR 1.3 4), respectively, $96.9 \%$ had HIV-1 RNA levels $<400$ copies $/ \mathrm{mL}$ at both time points. Of participants with available results, $75.0 \%$ and $76.7 \%$ had HIV-1 RNA levels $<50$ copies/mL within 12 months of treatment and at a median of 2 years (IQR 1.3 - 4), respectively, while only one had HIV-1 RNA levels of $\geq 400$ copies/mL at the two time points. The ERC received no reports of drug-related adverse events or deaths.

\section{Discussion}

This is the largest description of children and young adolescents treated with third-line ARVs in a public sector programme in SA. Based on the recommendations of the ERC, 34/35 children $(97.1 \%)$ received DRV/r, 25/34 (73.5\%) received RAL in addition to $\mathrm{DRV} / \mathrm{r}$, and only $3 / 34(8.8 \%)$ receiving $\mathrm{DRV} / \mathrm{r}$ received both $\mathrm{RAL}$ and ETR in addition (Table 2). Despite the study subjects having

Table 1. Characteristics of study participants $(N=35)$ receiving a DRV/r-, RAL- or ETR-containing ART regimen in the Western Cape

\begin{tabular}{ll} 
Province, SA & \\
\hline Characteristic & $8.8(5.5-11)$ \\
\hline Age (yr), median (IQR) & $15(42.9)$ \\
Female, $n$ (\%) & $2(5.7)$ \\
Year of 1st-line ART initiation, $n$ (\%) & $23(65.7)$ \\
Before 2004 & $7(20.0)$ \\
2004 - 2007 & $3(8.6)$ \\
2008 - 2011 & $6.9(5-9.9)$ \\
2012 - 2014 & \\
Years on ARVs prior to starting a DRV/r-, RAL- or ETR-containing ART regimen, median (IQR) \\
Number of ARVs exposed to prior to starting a DRV/r-, RAL- or ETR-containing ART regimen, \\
median (IQR) & $4(2-4)$ \\
NRTIs & $1(0-1)$ \\
NNRTIs & $1(1-2)$ \\
PIs & $13(37.1)$ \\
Previous exposure to unboosted PI (full-dose ritonavir)-containing 1st-line ART, $n(\%)$ & \\
ART treatment site at time of referral to ERC, $n$ (\%) & $13(37.1)$ \\
Primary care clinic & $3(8.6)$ \\
District hospital & $19(54.3)$ \\
Regional hospital/tertiary referral hospital & \\
Provincial distribution at time of referral to ERC, $n$ (\%) & $28(80.0)$ \\
Within Cape Town metropolitan area & $7(20.0)$ \\
Outside Cape Town metropolitan area & \\
Prior to starting a DRV/r-, RAL- or ETR-containing ART regimen, median $(\mathrm{IQR})$ & $405.5(251.5-541)(n=24)$ \\
CD4+ lymphocyte count (cells/ $\mu \mathrm{L})$ & $14.9(8.3-20.3)(n=13)$ \\
CD4+ lymphocyte percentage & $28314(5595.5-120186.5)(n=24)$ \\
HIV-1 RNA, copies/mL & $4.5(3.7-5.0)(n=24)$ \\
HIV-1 RNA, log & \\
\end{tabular}




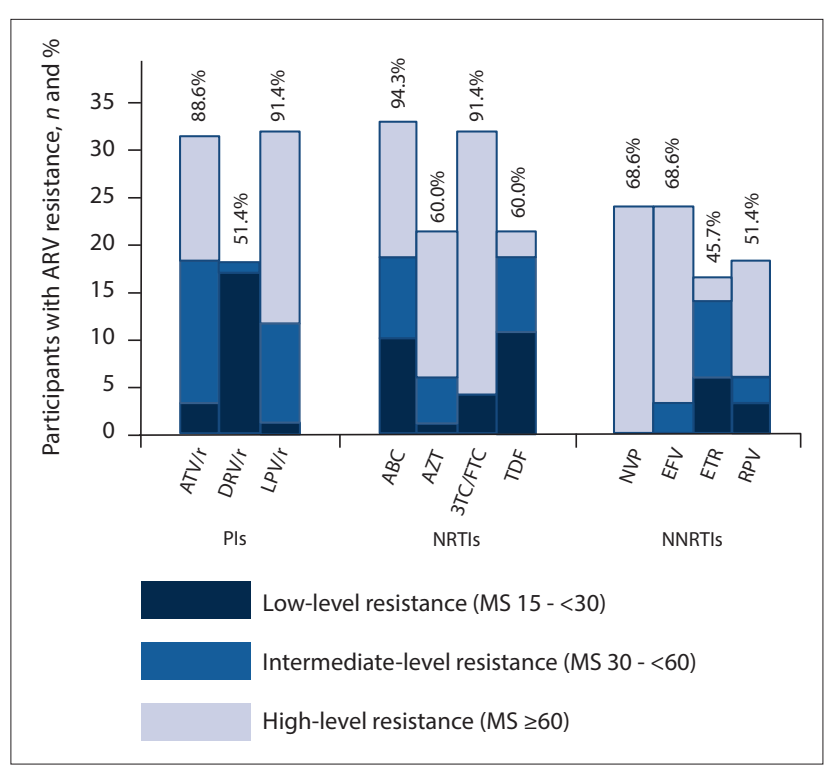

Fig. 1. Proportion of study subjects with resistance to selected ARV drugs prior to starting treatment on a DRV/r-, RAL- or ETR-containing antiretroviral therapy regimen. Drug resistance interpretation by mutation score category is according to the Stanford University HIV genotypic resistance interpretation algorithm performed at the time that the genotypic resistance test/s were done or, if not done then, at the time of application to the Expert Review Committee. $(A R V=$ antiretroviral $; D R V / r=$ darunavir/ritonavir; $R A L=$ raltegravir $; E T R=$ etravirine $; A T V / r=$ atazanavir/ritonavir $;$ $D R V / r=$ darunavir/ritonavir $[L P / r=$ lopinavir/ritonavir $; A B C=$ abacavir; $A Z T=$ zidovudine $; 3 T C / F T C=$ lamivudine/emtricitabine $T D F=$ tenofovi $;$ $N V P=$ nevirapine $; E F V=$ efavirenz; $R P V=$ rilpivirine $;$ PIs = protease inhibitors; NRTIs = nucleoside reverse transcriptase inhibitors; NNRTIs = non-nucleoside reverse transcriptase inhibitors; $M S=$ mutation score according to the Stanford University HIV genotypic resistance interpretation algorithm. $\left.{ }^{[17]}\right)$

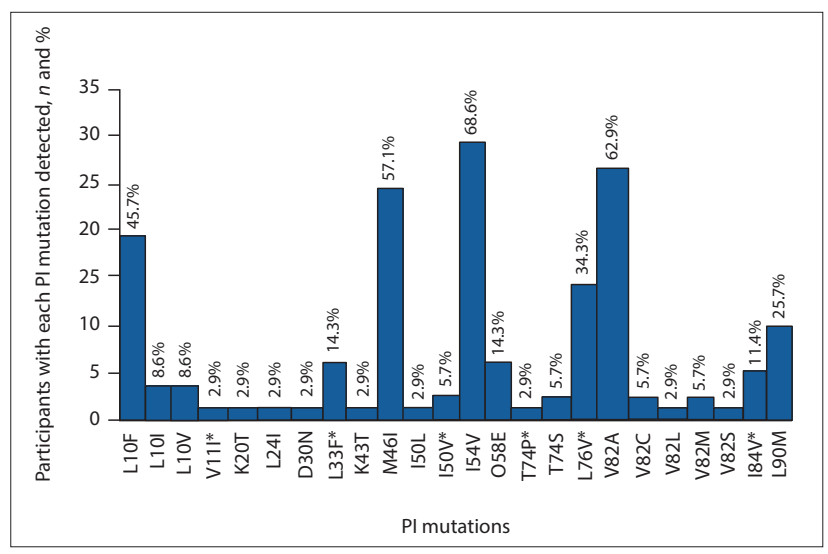

Fig. 2. Proportion of study subjects with each PI mutation detected prior to starting treatment on a DRV/r-, RAL- or ETR-containing ARV therapy regimen. $(P I=$ protease inhibitor; $D R V / r=$ darunavir/ritonavir; $R A L=$ raltegravir $; E T R=$ etravirine; $A R V=$ antiretroviral ${ }^{*} D R V$ resistanceassociated mutations.)

multidrug-resistant HIV prior to starting treatment with these regimens, including low-level DRV/r resistance in almost $50 \%$, we found impressive early treatment outcomes. Both within 12 months of treatment and after a median period of 2 years (IQR $1.3-4$ ) on treatment, $>96.5 \%$ of the participants with available results ( $86-91 \%$ at the two time points, respectively) had achieved HIV-1 RNA levels $<400$ copies/mL. Although significantly fewer CD4+ lymphocyte counts and percentage results were available both before and after starting a third-line regimen, the median $\mathrm{CD} 4+$ lymphocyte count of 717 cells $/ \mu \mathrm{L}$ (IQR 513 - 980) after a median of 2 years on treatment indicates good immunological reconstitution in participants with available results.

Just over half of the children received treatment with third-line ARV regimens at regional or tertiary hospitals, the remainder attending district-level hospitals or primary care clinics (Table 1). Decentralisation of care from large urban hospital settings to smaller rural hospitals and clinics is critically important in improving access to treatment for patients who may not be able to reach centralised healthcare facilities owing to financial and transport constraints. This is an important strength of this study.

It is significant that approximately a third of the participants (34.3\%) had received only one previous ART regimen (RTV- or LPV/r-based first-line ART, including single-drug substitutions from RTV to $\mathrm{LPV} / \mathrm{r}$, a single NRTI switch, or temporary 3TC monotherapy) before starting on the DRV/r-, RAL- or ETR-containing regimen, which was effectively a second-line ART regimen for these participants. In SA guidelines prior to 2010, the recommended second-line ART regimen for children or adolescents failing PI-based first-line ART was an NNRTI plus two NRTIs. ${ }^{[19]}$ In the current SA guidelines $(2014),{ }^{[20]}$ there is no standardised second-line treatment regimen for children failing first-line PI-based ART and no standardised third-line ART regimens. It is recommended that these children should be referred to an ERC so that treatment with third-line agents (including DRV/r, RAL and ETR) can be considered following interpretation of the GRT and taking into account prior ARV exposure. ${ }^{[20]}$ Although current WHO guidelines recommend a second-line regimen comprising two NRTIs plus either EFV or RAL for children failing first-line LPV/r-based ART, there is a lack of data to support these recommendations. ${ }^{[5]}$

The WHO guidelines do not define specific criteria for the inclusion of INSTIs, NNRTIs or NRTIs into third-line ART regimens. ${ }^{[5]}$ In a French cohort of 12 highly ARV-experienced adolescents all treated with a combination of DRV/r, RAL and ETR with additional ARVs in some patients, 11/12 (91.7\%) achieved HIV-1 RNA levels of $<400$ copies $/ \mathrm{mL}$ after a median of 12 months, with $6(50.0 \%)$ achieving $<50$ copies $/ \mathrm{mL}$. In addition, medication was generally well tolerated with no grade 3 or 4 adverse events reported and no treatment discontinuations. ${ }^{[6]}$ Other than phase 2 trials under controlled conditions, two other retrospective studies involving treatment-experienced adolescents in Spain receiving RALor ETR-based regimens in combination with other ARVs have found comparable safety and efficacy outcomes. . $^{[7,8,10-12]}$

Although GRT is a prerequisite for accessing these medications in the SA public sector, specific indications for resistance testing are not included in the 2014 national guidelines, and access to GRT has not been uniform in the SA public sector. Before 2015, GRT was generally only available to patients with private medical insurance or through research studies or donor-funded access programmes in the public sector. Since April 2015, access to GRT in the Western Cape public health sector has required written motivation by the clinician manging the patient and approval by the provincial Department of Health. Patients on a PI-based ART regimen with HIV-1 RNA non-suppression (defined as at least three HIV-1 RNA measurements of $\geq 1000$ copies $/ \mathrm{mL}$ ( $\geq \log 4.5$ ) at least 8 - 12 weeks apart), with the most recent HIV-1 RNA level result within $3-6$ months of the application, are eligible for GRT. In addition, children and early adolescents ( $<15$ years of age) must have been receiving a PI-based 


\begin{tabular}{|c|c|c|}
\hline ART regimen & Children, $n$ (\%) & \\
\hline $\mathrm{DRV} / \mathrm{r}+2$ NRTIs & $9(25.7)$ & \\
\hline $\mathrm{DRV} / \mathrm{r}+\mathrm{RAL}+1 \mathrm{NRTI}$ & $8(22.8)$ & \\
\hline $\mathrm{DRV} / \mathrm{r}+\mathrm{RAL}+2$ NRTIs & $14(40.0)$ & \\
\hline $\mathrm{DRV} / \mathrm{r}+\mathrm{RAL}+\mathrm{ETR}+1 \mathrm{NRTI}$ & $3(8.6)$ & \\
\hline $\mathrm{ETR}+\mathrm{LPV} / \mathrm{r}+1 \mathrm{NRTI}$ & $1(2.9)$ & \\
\hline Treatment duration & Years, median (IQR) & \\
\hline Duration of DRV/r-, RAL- or ETR-containing ART at time of analysis & $2(1.3-4)$ & \\
\hline Outcomes & Within 12 months & At time of analysis \\
\hline CD4+ count $($ cells $/ \mu \mathrm{L})$, median (IQR) & $\begin{array}{l}649(506-900) \\
(n=23)\end{array}$ & $\begin{array}{l}717(513-980) \\
(n=21)\end{array}$ \\
\hline CD4+ lymphocyte percentage, median (IQR) & $\begin{array}{l}27.1(20.8-36.4) \\
(n=23)\end{array}$ & $\begin{array}{l}27.2(20.8-35.6) \\
(n=21)\end{array}$ \\
\hline HIV-1 RNA (copies/mL), $n(\%)$ & $(n=32)$ & $(n=30)$ \\
\hline$<50$ & $24(75.0)$ & $23(76.7)$ \\
\hline$<400$ & $31(96.9)$ & $29(96.7)$ \\
\hline$\geq 400$ & $1(3.1)$ & $1(3.3)$ \\
\hline
\end{tabular}

ART regimen for at least 1 year, and adults and late adolescents ( $\geq 15$ years of age) must have been receiving a PI-based ART regimen for at least 2 years. ${ }^{[18]}$

An important factor that may have contributed to the development of significant PI resistance in the study subjects is failure to have achieved viral suppression while on treatment with an unboosted PI-based ART regimen. Such regimens were previously used to treat children $<6$ months of age and children requiring concomitant rifampicin (RIF)-based antituberculosis treatment. Unboosted PIs are known to be associated with the development of major PI resistance mutations in children with virological failure. ${ }^{[21-23]}$ In our study, $13 / 35$ subjects ( $37.1 \%$ ) had received previous treatment with a RTV-based regimen. Pharmacokinetic interactions between RIF and LPV/r resulting in subtherapeutic plasma LPV levels may also predispose to the development of PI resistance. ${ }^{[24-26]}$ Adjustment of first- or second-line ART regimens to avoid this problem may not have been done in all cases. However, detailed information on TB co-infection and treatment was not included in this study.

The presence of high rates of NNRTI resistance mutations conferring resistance to ETR in nearly $50 \%$ of subjects is likely to reflect virological failure on first- or second-line NNRTI-based ART regimens. However, genotyping is not routinely performed at the time of first-line ART failure, and this may be an underestimation of the true prevalence of NNRTI mutations conferring resistance to ETR. Once the patient is no longer receiving NNRTI-based ART, some mutations may remain present at low levels not detected by routine genotyping done at the time of second-line ART failure. Previous studies have described high-level ETR resistance in almost $50 \%$ of children failing first-line NNRTI-based ART. ${ }^{[27,28]}$ These findings call into question the role of ETR in second- and third-line ART regimens in NNRTI-exposed individuals.

Although adult and some paediatric formulations of DRV, RAL and ETR are registered in SA, these are often inappropriate for young children, in whom doses must be adjusted for age and weight changes. Young children who are unable to swallow tablets require dispersible or chewable tablets or oral suspensions. Although RAL chewable tablets are now available in SA, oral suspensions of DRV, RAL and ETR are not registered here and are only available on compassionate access programmes from manufacturers and with approval from the Medicines Control Council of SA. In addition, $\mathrm{DRV} / \mathrm{r}$ is not currently available as a co-formulated medication, so RTV needs to be provided separately, adding to the pill burden of third-line regimens. Access to and palatability of RTV oral suspension remains a significant barrier to third-line ART for younger children.

\section{Study limitations}

This study has a number of limitations related to its retrospective nature and the fact that participants were managed at multiple healthcare facilities across the province without direct oversight by members of the ERC. We were unable to obtain complete data on HIV-1 RNA levels and CD4+ lymphocyte counts on all study participants both before and after they started their DRV/r-, RAL- or ETR-containing ART regimens. Some participants had started these regimens months or years prior to being reviewed by the ERC, and the data were not always supplied by the referring clinicians or could not be traced from an earlier electronic laboratory reporting system. Since this was not a prospective study, monitoring of HIV-1 RNA levels, CD4+ lymphocyte counts and adverse events may not always have been performed according to standard provincial monitoring guidelines. Information regarding maternal ART and exposure of participants to prevention of mother-to-child transmission of HIV would have contributed to an understanding of participants with NNRTI mutations in whom there was no history of previous NNRTI-based ART regimens. The children and adolescents reviewed by the ERC and started on third-line ART regimens may not be representative of other children who are failing first- or secondline ART in other regions and who may not have undergone genotyping. Documentation of prior treatment with unboosted PI-based ART and prior concomitant PI-based ART treatment and antituberculosis treatment was common, but data were not available for all participants. This study involved a small number of participants receiving individualised ARV combinations based on current and historical genotype results and prior ARV history interpreted by an ERC and is not able to compare the relative effectiveness of the different treatment regimens used. 


\section{Conclusions}

This study found DRV/r-, RAL- or ETR- containing ART regimens to be effective in a group of ART-experienced children and adolescents with multidrug-resistant HIV. Although the ART regimens in this study were individualised based on genotyping results, further research evaluating the safety and efficacy of standardised thirdline treatment regimens in children of all ages and adolescents is needed in order to improve access in settings where genotyping is not routinely available.

Acknowledgements. Members of the paediatric ERCs: National: L Levin, M Archary, P Jeena, A Coovadia, J Nuttall, K Jamaloodien, N Jagaroo; provincial: M Cotton, H Rabie, B Eley, M Hsiao, J Nuttall, J Voget.

Author contributions. JN and VP conceived, designed and obtained ethical approval for the study, developed the study database, analysed the data, and prepared and approved the manuscript for submission.

Funding. None.

Conflicts of interest. None.

1. United Nations Children's Fund. Towards an AIDS-Free Generation - Children and AIDS: Sixth Stocktaking Report, 2013. New York: UNICEF 2013, https://www wniceforo/publications/file/ Children_and_AIDS_Sixth_Stocktaking Report_EN.pdf (accessed 30 April 2017).

Joint United Nations Programme on HIV/AIDS. 2013 Progress Report on the Global Plan Towards the

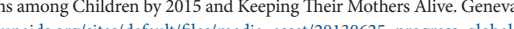
UNAIDS, June 2013. http://www.unaids.org/sites/default/files/media_asset/20130625_progress_global_
plan_en_0.pdf (accessed 30 April 2017).

World Health Organization. Antiretroviral Medicines in Low- and Middle-income Countries: Forecast of Global and Regional Demand for 2014 - 2018 (technical report). Geneva: WHO, July 2015. http:/ www.who.int/hiv/pub/amds/arv-forecast2014-2018/en/ (accessed 30 April 2017).

4. Lazarus E, Nicol S, Penazzato M, Cotton M, Tablante E, Violari A. Second and third line antiretrovira therapy options for children and adolescents: A systematic review. Presented at the 7th Internationa Workshop on HIV Pediatrics, Vancouver, Canada, 17 - 18 July 2015. Abstract 23. http://regist2.virologyeducation.com/abstractbook/2015_8.pdf (accessed 30 April 2017).

5. World Health Organization. Consolidated Guidelines on the Use of Antiretroviral Drugs for Treating an Preventing HIV Infection: Recommendations for a Public Health Approach. 2nd ed. WHO, 2016. http:// www.who.int/hiv/pub/arv/arv-2016/en/ (accessed 30 April 2017).

6. Thuret I, Chaix M-L, Tamalet C, et al. Raltegravir, etravirine and $\mathrm{r}$-darunavir combination in adolescents with multidrug-resistant virus. AIDS 2009:23(17):2364-2366, https://doi.org/10.1097/ gad. 0 b013e3283310456

7. Blanche S, Bologna R, Cahn P, et al. Pharmacokinetics, safety and efficacy of darunavir/ritonavir in Blanche S, Bologna R, Cahn P, et al. Pharmacokinetics, safety and efficacy of darunavir/ritonavir in
treatment-experienced children and adolescents. AIDS 2009;23(15):2005-2013. https://doi.org/10.1097 gad.0b013e328330abaa

8. Violari A, Bologna R, Kumarasamy N, et al. Safety and efficacy of darunavir/ritonavir in treatment-experienced pediatric patients: Week 48 results of the ARIEL trial. Pediatr Infect Dis J 2015:34(5):e132-e137. htt

9. Flynn P, Komar S, Blanche S, et al. Efficacy and safety of darunavir/ritonavir at 48 weeks in treatmentnaive, HIV-1-infected adolescents: Results from a phase 2 open-label trial (DIONE). Pediatr Infect Dis 2014;33(9):940-945. https://doi.org/10.1097/inf.0000000000000308
10. Briz V, Palladino C, Navarro M, et al. Etravirine-based highly active antiretroviral therapy in HIV1-infected paediatric patients. HIV Med 2011;12(7):442-446. https://doi.org/10.1111\%2Fj.14681-infected paedia

11. Tudor-Williams G, Cahn P, Chokephaibulkit K, et al. Etravirine in treatment-experienced, HIV-1infected children and adolescents: 48-week safety, efficacy and resistance analysis of the phase II PIANO study. HIV Med 2014;15(9):513-524. https://doi.org/10.1111\%2Fhiv.12141

12. Briz V, Leon-Leal JA, Palladino C, et al. Potent and sustained antiviral response of raltegravir-based highly active antiretroviral therapy in HIV type 1-infected children and adolescents. Pediatr Infect Dis J 2012;31(3):273-277. https://doi.org/10.1097/INF.0b013e31824580e8

13. Nachman S, Zheng N, Acosta EP, et al. Pharmacokinetics, safety, and 48-week efficacy of oral raltegravir in HIV-1-infected children aged 2 through 18 years. Clin Infect Dis 2014;58(3):413-422. https://doi. org/10.1093\%2Fjpids\%2Fpiul46

14. Kindra G, Sipambo N, Moultrie H, Fairlie L. Outcomes in treatment with darunavir/ritonavir in ARTexperienced paediatric patients. S Afr Med J 2015;105(5):330-331. https://doi.org/10.7196\%2FSAMI.9211

15. Panel on Antiretroviral Therapy and Medical Management of HIV-Infected Children. Guidelines for the Use of Antiretroviral Agents in Pedintric HIV Infection. http://aidsinfonih gov/contentfiles/lvguidelines/ pediatricguidelines.pdf (accessed 23 March 2017).

16. Full US Prescribing Information for Tivicay. https://www.gsksource.com/pharma/content/dam/ GlaxoSmithKline/US/en/Prescribing_Information/Tivicay/pdf/TIVICAY-PI-PIL.PDF (accessed $30 \mathrm{April}$
(1) 2017).

17. Stanford University HIV drug resistance database. https://hivdb.stanford.edu/ (accessed 30 April 2017).

18. Provincial Government of the Western Cape, South Africa. The Western Cape Consolidated Guidelines for HIV Treatment: Prevention of Mother-to-Child Transmission of HIV (PMTCT), Children, Adolescents and Adults. HIV/AIDS/STI/TB (HAST) Directorate, Department of Health, Provincial Government of the Western Cape, April 2015. http://www.paediatrics.uct.ac.za/sites/default/files/image_ tool/images/38/Western\%20Cape\%20Consolidated\%20HIV\%20guidelines\%20November\%202015.pdf (accessed 30 April 2017)

19. Meyers T, Eley B. Guidelines for the management of HIV-infected children (National Department of Health, South Africa 2005). South Afr J HIV Med 2005;6(4):33. https://doi.org/10.4102\%2Fsajhivmed. v6i4.580

20. National Department of Health, South Africa. National Consolidated Guidelines for the Prevention of Mother-to-child Transmission of HIV (PMTCT) and the Management of HIV in Children, Adolescents and Adults. Pretoria: NDoH, December 2014. http://www.health.gov.za/index.php/2014-03-17-09-0938/policies-and-guidelines/category/230-2015p\# (accessed 30 April 2017).

21. Van Zyl G, van der Merwe L, Claassen M, et al. Protease inhibitor resistance in South African children with virologic failure. Pediatr Infect Dis J 2009;28(12):1125-1127. https://doi.org/10.1097\%2Finf.0b013 e318lafs29d

22. Taylor BS, Hunt G, Abrams EJ, et al. Rapid development of antiretroviral drug resistance mutations in HIV-infected children less than two years of age initiating protease inhibitor-based therapy in South Africa. AIDS Res Hum Retroviruses 2011;27(9):945-956. https://doi.org/10.1089\%2Faid.2010.0205

23. Van Zyl G, Rabie H, Nuttall J, Cotton M. It is time to consider third-line options in antiretroviral-experienced paediatric patients? J Int AIDS Soc 2011;14(1):55. https://doi.org/10.1186\%2F1758-2652-14-55

24. Ren Y, Nuttall J, Egbers C, et al. Effect of rifampicin on lopinavir pharmacokinetics in HIV-infected children with tuberculosis. J Acquir Immune Defic Syndr 2008;47(5):566-569. https://doi.org/10.1097 \%2Fqai.0b013e3181642257

25. La Porte C, Colbers E, Bertz R, et al. Pharmacokinetics of adjusted dose lopinavir-ritonavir combined with rifampicin in org/10.1128\%2Faac. 48.5.1553-1560.2004

26. Mcilleron $\mathrm{H}$, Ren $\mathrm{Y}$, Nuttall J, et al. Lopinavir exposure is insufficient in children given double doses of lopinavir/ritonavir during rifampicin-based treatment for tuberculosis. Antivir Ther 2011;16(3):417-421. https://doi.org/10.3851\%2Fimp1757

27. Puthanakit T, Jourdain G, Hongsiriwon S, et al. HIV-1 drug resistance mutations in children after failure of first-line nonnucleoside reverse transcriptase inhibitor-based antiretroviral therapy. HIV Med 2010;11(9):565-572. https://doi.org/10.1111\%2Fj.1468-1293.2010.00828.x

28. Steegen K, Bronze M, Papathanasopoulos MA, et al. HIV-1 antiretroviral drug resistance patterns in patients failing NNRTI-based treatment: Results from a national survey in South Africa. J Antimicrob Chemother 2017;72(1):210-219. https://doi.org/10.1093\%2Fjac\%2Fdkw358

Accepted 14 August 2017. 